\title{
Impact of Therapeutic Hypothermia During Cardiopulmonary Resuscitation on Neurologic Outcome: A Systematic Review and Meta-analysis
}

Filippo Annoni ( $\nabla$ annonifilippo@gmail.com )

Department of Intensive Care Erasme Hospital, Free University of Brussels (ULB) Lennik road, 8081070 BRUSSELS, Belgium https://orcid.org/0000-0002-1271-9034

Lorenzo PELUSO

Erasme Hospital: Hopital Erasme

Marco FIORE

Erasme Hospital: Hopital Erasme

\section{Per NORDBERG}

Karolinska Institute: Karolinska Institutet

\section{Leif SVENSSON}

Karolinska Institute: Karolinska Institutet

\section{Benjamin ABELLA}

University of Pennsylvania Perelman School of Medicine

\section{Lorenzo CALABRO'}

Erasme Hospital: Hopital Erasme

\section{Sabino SCOLLETTA}

Siena University Hospital: Azienda Ospedaliera Universitaria Senese

\section{Jean-Louis VINCENT}

Erasme Hospital: Hopital Erasme

Jacques CRETEUR

Erasme Hospital: Hopital Erasme

\section{Fabio Silvio TACCONE}

Erasme Hospital: Hopital Erasme

\section{Research}

Keywords: Cardiac arrest, intra-arrest hypothermia, CPR, cryotherapy

Posted Date: October 8th, 2020

DOl: https://doi.org/10.21203/rs.3.rs-86996/v1 
License: (c) (i) This work is licensed under a Creative Commons Attribution 4.0 International License. Read Full License

Version of Record: A version of this preprint was published at Resuscitation on May 1st, 2021. See the published version at https://doi.org/10.1016/j.resuscitation.2021.01.029. 


\section{Abstract}

Background: Therapeutic cooling initiated during cardiopulmonary resuscitation (intra arrest therapeutic hypothermia, IATH) provided diverging effect on neurological outcome of out-of-hospital cardiac arrest (OHCA) patients depending on the initial cardiac rhythm and the cooling methods used.

Methods: We performed a systematic search of PubMed, EMBASE and the CENTRAL databases using established Medical Subject Headings (MeSH) terms for IATH and OHCA. Only studies comparing IATH to standard in-hospital targeted temperature management (TTM - control group) were selected. We used the revised Cochrane RoB-2 and the Newcastle-Ottawa scale tool to assess risk of bias of each study. Primary outcome was favorable neurological outcome (FO); secondary outcomes included return of spontaneous circulation (ROSC) rate and overall survival to hospital discharge. Two authors independently assessed the validity of included human studies and extracted data regarding characteristics of the studied cohorts and main outcomes.

Results: Out of 20950 studies, 8 studies ( $n=3493$ patients, including 4 randomized trials, RCTs) were included in the final analysis. When compared to controls, the use of IATH was not associated with improved favorable neurological outcome (OR 0.96 [95\% Cls 0.68-1.37]; $p=0.84$ ), increased ROSC rate (OR 1.11 [95\% Cls 0.83-1.49]; $p=0.46$ ) or survival to hospital discharge (OR 0.91 [95\% Cls 0.73-1.14]; $p=$ $0.43)$. Significant heterogeneity among studies was observed only for the analysis of ROSC rate $(R=69 \%)$. Trans-nasal evaporative cooling and cold fluids were explored in two RCTs each and no significant differences were observed on neurological outcome. However, trans-nasal evaporative cooling was associated with a higher probability of favorable neurological outcome when compared to controls in patients with an initial shockable rhythm (OR 1.62 [95\% Cl 1.00-2.64]; $p=0.05]$.

Conclusions: In this meta-analysis, IATH was not associated with improved neurological outcome when compared to standard in-hospital TTM. However, there are considerable outcome differences depending on the methods used and the studied population that need to be explored in future trials.

\section{Clinical Trial Registration: PROSPERO (CRD42019130322)}

\section{Introduction}

Anoxic brain injury frequently causes significant neurological sequelae and is the main cause of poor outcome in resuscitated cardiac arrest patients $(C A)[1,2]$. At present, there are few established treatments that in randomized trials have shown to limit the magnitude of such injury. Thus, these patients in general receive supportive therapy and admission to the intensive care units (ICUs) to control the systemic causes of secondary brain damage (i.e. fever, anemia, hypocapnia, hyponatremia, hyperoxemia and dysglycemia).

The use of targeted temperature management (TTM) with a core body temperature of $33-36^{\circ} \mathrm{C}$ for at least 24 hours is recommended to mitigate brain injury. [3]. In particular, rapid brain cooling has been 
considered to have the greatest potential to reduce the extent of post-anoxic brain injury in the immediate post-CA phase, through different mechanisms, including reduction of cerebral metabolic rate, excitatory amino acids release, reduction in inflammation and apoptotic signals and free radical production [4-7].

However, diverse aspects related to the application of TTM remain controversial, including the optimal target temperature, patient selection, timing of TTM initiation, the most effective methods to induce and maintain TTM, the duration of the cooling phase and the rewarming rate/fever control strategies [8]. In particular, contrary to cardiac arrest laboratory investigations, initiation of TTM is often delayed by several hours in clinical practice because of several factors, such as the need for transportation or diagnostic procedures, the availability and performance of different cooling devices, thus potentially limiting its beneficial effects. To reduce the delay between the onset of the cardiac arrest and TTM initiation, several trials has focused on the early pre-hospital period and provision of TTM in the field, i.e. either during cardiopulmonary resuscitation (CPR) or immediately after ROSC prior to hospital arrival. This very early approach, which has been identified as "intra-arrest therapeutic hypothermia" (IATH), has been administered by different cooling methods, including selective brain cooling, rapid infusion of cold fluids and trans-nasal evaporative cooling (TNEC) [9-16]. However, despite some preliminary encouraging findings [10], variable results in RCTs have suggested either a potential benefit (i.e. in OHCA patients with an initial shockable rhythm) or deleterious effects, in particular when large volumes of cold fluids have been used [14, 16-17].

The aim of this systematic review and meta-analysis was therefore to investigate whether the application of IATH could have an impact on clinically relevant outcomes, including neurological outcome (FO), return of spontaneous circulation (ROSC) and overall survival, in patients suffering from out-of-hospital cardiac arrest (OHCA).

\section{Methods}

We adhered to the Preferred Reporting Items for Systematic Reviews and Meta-Analysis - Protocols (PRISMA-P) guidelines [18]. The protocol of this study was registered with the International Prospective Register of Systematic Reviews (PROSPERO) and last edited on the 2nd of June 2020 (CRD42019130322).

\section{Data sources and Search strategies}

A systematic literature search was performed up to the 28th of May 2020 in the PubMed, EMBASE and CENTRAL databases using the following Medical Subject Headings (MeSH) terms: "heart arrest" AND/OR "hypothermia" AND/OR "hypothermia, induced" AND/OR cryotherapy" AND/OR cardiopulmonary resuscitation" AND/OR critical care outcomes" AND/OR "patient outcome assessment" AND/OR "morbidity" AND/OR "mortality". Only studies using IATH and having a control group treated with standard in-hospital care including TTM were selected for the final analysis. 
The search included only original studies published in English in peer-reviewed journals. The complete research string for each database as well as the main research questions, with reference to participants, interventions, comparisons, outcomes and study design (PICOS), are reported in ESM (Appendix 1 and Table S1). In addition, we searched the reference lists of eligible studies and relevant reviews for additional published and unpublished data, searched by contacting experts, and used a web search for abstracts, proceedings, and unpublished studies.

\section{Study screening and selection}

Two authors (FA and LP) independently screened study titles and abstracts for potential eligibility and assessed their validity. Disagreement between authors was assessed and resolved through a third reviewer (FST), who reviewed the original text of the article. FA and MF extracted data regarding characteristics of the studied cohort and the main outcomes related to the use of IATH including neurological outcome, ROSC rate and survival.

In the analysis, we included only studies evaluating IATH in adult ( $>18$ years of age) CA patients in either retrospective, prospective or randomized controlled trials (RCTs). Studies conducted in healthy volunteers or in animal models were excluded. Editorials, commentaries, letters to editor, opinion articles, reviews, meeting abstracts, case reports, and studies published in other languages were also excluded, as well as original articles lacking abstract and/or quantitative details on neurological outcome and survival. When multiple publications of the same research group/center described case series with potential overlap, the more recent publication, if eligible, was considered. None of the authors of the original studies was contacted to obtain further information, which were not available in the published manuscript. Only studies that met all the above criteria were incorporated for quantitative synthesis.

\section{Appraisal of study quality}

The level of evidence (LOE) of each study was assessed according to the Grading of Recommendations, Assessment, Development and Evaluations (GRADE) evidence system [19]. For RCTs, the risk of bias (ROB) was assessed using the Revised Cochrane RoB-2 tool that classifies the ROB as "low," "probably low," "probably high," or "high" for each of the following domains: randomization process, deviation from the intended interventions, missing outcomes data, measurement of the outcome and selection of the reported results. [20] The ROB for non-RCTs was assessed using the Newcastle-Ottawa Scale [21]; three different components were evaluated for each study: (a) selection of cases: studies were considered as "low" ROB if case definition was adequate, cases were representative and outcome of interest was not present at the beginning of the study; (b) comparability of cohorts: studies were considered as "Iow" ROB if adjustment was made for usual prognostic factors (i.e., Utstein variables); (c) exposure and outcome: studies were considered as "low" ROB if assessment of outcome and follow-up were appropriate. Overall, a study was considered as "low" ROB if each single component was classified as "low." LOE was further analyzed by two experts (FST, PN) and one independent statistician. Disagreement was resolved by consensus. 
The primary outcome of the meta-analysis was the occurrence of favorable neurological outcome (FO), whenever it was recorded. Favorable neurological outcome was defined as a Cerebral Performance Category of 1-2. Secondary outcomes were ROSC rate and survival, whenever this was collected. Predefined analyses were performed in subgroups of patients: a) IATH using TNEC vs. IATH using other methods; b) patients presenting with an initial shockable rhythm.

\section{Statistical analysis}

Means of survival and favorable neurological outcomes probabilities were obtained by weighting each study by the inverse of variance. The Mantel-Haenszel method was chosen as the reference method for fixed effects analysis. The Mantel-Haenszel formula is applied to calculate an overall, unconfounded, effect estimate of a given exposure for a specific outcome by combining stratum-specific odds-ratios (OR). Stratum-specific ORs are calculated within each stratum of the confounding variable and compared with the corresponding effect estimates in the whole group. A $Z$ test was carried out to assess the significance of the risk differences. The $R$ was calculated by $\chi^{2}$ test to assess variability due to heterogeneity rather than chance. A substantial heterogeneity was assumed with $R>50 \%$. 95\% confidence intervals (Cls) for neurologic outcome and survival were calculated with the Wilson method and placed in forest plots and statistical significance was assumed for $p<0.05$. The presence of publication bias was evaluated by trim and fill. The trim and fill method estimates the number of missing studies from a meta-analysis due to the suppression of the most extreme results on one side of the funnel plot. Then, this method augments the observed data and recomputes the summary estimate based on the complete data. The trim and fill outputs were obtained with iterations. Analyses were performed for all the selected studies, as well as grouped by RCT vs. observational trials. Statistical analysis was conducted by Review Manager 5.3 software and funnel and forest plots were developed.

\section{Results}

\section{Study selection}

A total of 20950 records were identified after the initial search. After the first screening procedure, 43 studies were assessed for eligibility. Of those, 35 did not fit inclusion criteria, as reported in Figure 1; a total of 8 studies [9-16], including 3493 patients, were then included for meta-analysis.

\section{Study characteristics}

The characteristics of the selected studies are summarized in Table 1. We identified 4 RCTs (high quality of evidence for two $[12,16]$ and moderate for two $[10,14]$ ), two prospective studies (low level of evidence in one [9] and very low quality of evidence [15] in the other, which compared a prospective cohort with historical controls) and two retrospective studies (very low quality of evidence [11,13]). The ROB for RCTs was low in three studies [10,12,16] and showed some concerns in one [14] (Table 2). For all non-RCTs, the ROB was high $[9,11,13,15]$ (Table 3 ). All selected studies included only patients after OHCA. In five 
studies, IATH was induced using intravenous cold fluids [11-15], whereas two studies used TNEC [10,16] and one selective cranial cooling [9].

Analysis of outcomes in all studies

Favorable neurological outcome was defined in 7 studies $(n=2945)$ as CPC of 1-2 [9-11, 13-16] recorded either at hospital discharge $(n=4,57.1 \%)$ or at 90 days $(n=2,28.5 \%)$ as well as discharge home in one study $(n=1,14.2 \%)$ [14]. ROSC rate was available in all studies $(n=3358)$ [9-16]. All studies also reported survival rate $(n=3487)$ [9-16], mostly registered at hospital discharge [9-15].

Overall, patients treated with IATH showed a similar probability for FO than controls (OR 0.96 [95\% Cls 0.68-1.37]; $p=0.84$, Figure 2A); however, there was a higher probability for $F O$ in the control group when only non-RCTs were considered (OR=0.56 [95\% Cls 0.32-0.96]; $p=0.03)$, while a non-significant trend in favor of IATH was observed in RCTs.

Patients treated with IATH showed a similar probability for both ROSC rate (OR 1.11 [95\% Cls 0.83-1.49]; $p=0.46$, Figure $2 B$ ) and survival (OR 0.91 [95\% Cls 0.73-1.14]; $p=0.43$, Figure $2 C$ ) than controls. No significant heterogeneity was observed among studies for $F O$ and survival analysis $(R=43 \%$ and $R=15 \%$, respectively); a significant heterogeneity was observed among studies for ROSC rate $(R=69 \%)$.

Analysis of outcomes according to the cooling methods

Two RCTs explored TNEC $[10,16]$ and reported favorable neurological outcome, ROSC rate and survival rate ( $n=430$ for IATH and 435 for controls); two other RCTs reported favorable neurological outcome, ROSC rate and survival rate ( $n=741$ for IATH and 702 for controls) for intravenous cold fluids [12,14]. Patients treated with IATH showed overall a similar probability for FO (OR=1.19 [95\% Cls 0.90-1.56]; $p=$ 0.21 , Figure 3), ROSC rate (Figure S1) and survival (Figure S2), regardless of the used IATH technique.

\section{Analysis of outcomes according to initial rhythm}

Specific data on neurological outcome according to the initial rhythm were reported only in two studies $[10,16]$. Among patients with an initial shockable rhythm, FO occurred more frequently in the IATH than in the control group (OR=1.62 [95\% Cls 1.00-2.64]; $p=0.05$, Figure 4); no differences in patients with an initial non-shockable rhythm were observed. Three studies reported data on survival rate according to the initial rhythm $[10,14,16]$; no differences in ROSC rate and survival were observed between IATH and controls, regardless of the initial rhythm (Figure S3).

\section{Discussion}

This systematic review and meta-analysis analyzes the application of IATH in the clinical setting, including 8 studies and 3493 patients. Our results showed that IATH does not improve the occurrence of favorable neurological outcome, ROSC rate or survival in the entire population when compared to standard in-hospital care. Nevertheless, it also appeared that specific subgroups of patients, such as the 
ones presenting with an initial shockable rhythm, might benefit the most from such intervention. Moreover, the method selected to induce IATH might also be relevant, suggesting a possible greater benefit from the use of TNEC compared to other methods.

Previously, other reviews and meta-analysis have been conducted to explore the impact of pre-hospital cooling after cardiac arrest [22-27], but none of them was specifically designed to investigate the application of only IATH. To our knowledge, only a previous review conducted in 2012 [28] specifically addressed this topic, but human data were scarce at the time, with the two largest published trials missing $[14,16]$, and also experimental studies were included. Two additional studies investigating IATH using cold intravenous fluids in the human setting, for a total of 50 patients, were also identified during the research process but were eventually excluded because of the lack of a control group $[29,30]$. The two largest studies included, despite not showing significant effects of IATH, suggested opposite effects of the treatment regarding both ROSC and survival rate. In the study by Bernard et al. [14], IATH was induced using rapid infusion of cold intravenous fluids and the trial was stopped after enrolling $48 \%$ of the previewed recruiting target due to changes in TTM target in most Australian sites. In this trial, patients treated with IATH required longer CPR, additional epinephrine, experienced more frequently pulmonary edema on admission and, among patients with an initial shockable rhythm, a significantly lower ROSC rate was reported. Interestingly, in the study by Nordberg et al. [16], which used TNEC to induce IATH, no differences were observed between groups related to the ROSC rate, adrenaline requirements and CPR duration. However, a higher proportion of patients achieving FO, in particular complete neurological recovery (i.e. CPC 1) was observed in the IATH group when patients with an initial shockable rhythm were analyzed. Whether the method used to induce IATH could explain those differences remains debatable, but our findings suggest that possibly TNEC might be more effective in patients presenting with shockable rhythm, probably because of selected and rapid brain cooling and the lack of adverse effects on the coronary perfusion pressure, which can be reduced by cold fluids [31].

Our study has several limitations. First, despite not being significant in most of the performed analyses, the heterogeneity between studies regarding differences in IATH protocol, including the timing to assess the outcome, have probably reduced the possibilities to identify an effect of the treatment. Second, we could not exclude that differences in managing neuro-prognostication and withdrawal of life sustaining therapies would result in diverse end-of life procedures among the selected trials, possibly influencing the pooled results. Third, the number of patients that could be included for subgroup analysis was limited and our results are only hypothesis-generating. Forth, no patient data were available, thus introducing potential limitations to adjust for confounders. Fifth, TTM protocols may vary between hospitals and along the time, possibly modifying the post-resuscitation care and thus having an impact on the neurological outcome of the patients included. Finally, several studies presented with a high risk of bias or raising some concerns, thereby reducing the robustness of our findings.

\section{Conclusions}


This systematic review and meta-analysis showed that IATH does not improve the occurrence of favorable neurological outcome, ROSC rate or survival when compared to standard in-hospital TTM in the global population of OHCA patients, but it could possibly be beneficial in the subgroup of patients presenting with a shockable rhythm if treated with TNEC. Further studies should then assess how to identify the population of patients that might benefit the most from such intervention.

\section{Abbreviations}

IATH

Intra arrest therapeutic hypothermia

OHCA

out-of-hospital cardiac arrest

$\mathrm{MeSH}$

medical subject headings

TTM

target temperature management

FO

favorable outcome

ROSC

return of spontaneous circulation

RCTs

randomized controlled trials

CA

cardiac arrest

CPR

cardiopulmonary resuscitation

TNEC

trans-nasal evaporative cooling

PRISMA-P

preferred reporting items for systematic reviews and meta-analysis-protocols

LOE

level of evidence

GRADE

grading of recommendations, assessment, development and evaluation

ROB

risk of bias

OR

odds-ratio

Cls

confidence intervals 
CPC

cerebral performance category

\section{Declarations}

Ethics approval and consent to participate. not applicable.

Consent for publication. not applicable.

Availability of data and materials. All data generated or analyzed during this study are included in this published article (and its supplementary information files).

Competing interests

Fabio Silvio TACCONE and Benjamin ABELLA received lecture fees from BD. Fabio Silvio TACCONE also received lecture fees from ZOLL

\section{Source of funding}

Dr Filippo Annoni was supported by the Fonds Erasme pour la Recherche Médicale

\section{Authors' contribution}

FA, FST and MF have generated the research string used for database interrogation.FA and LP independently screened study titles and abstracts for potential eligibility and assessed their validity. Disagreement between authors was assessed and resolved through a third reviewer (FST), who reviewed the original text of the article. FA and MF extracted data regarding characteristics of the studied cohort and the main outcomes related to the use of IATH including neurological outcome, ROSC rate and survival. FA and FST have produced a first draft of the manuscript and PN, LS, BA, LC, SS, JLV and JC have substantially revised the manuscript. All the authors have approved the final version of the manuscript and agreed on the submission of the work for pubblication.

\section{References}

1. Executive summary. 2015 International consensus on cardiopulmonary resuscitation and emergency cardiovascular care science with treatment recommendations. Resuscitation. 2015;95:e1-31.

2. Lemiale $\mathrm{V}$, Dumas $\mathrm{F}$, Mongardon $\mathrm{N}$, et al. Intensive care unit mortality after cardiac arrest: the relative contribution of shock and brain injury in a large cohort. Intensive Care Med. 2013;39:1972-80.

3. Taccone FS, Crippa IA, Dell'Anna AM, Scolletta S. Neuroprotective strategies and neuroprognostication after cardiac arrest. Best Pract Res Clin Anaesthesiol. 2015;29:451-64.

4. Erecinska M, Thoresen M, Silver IA. Effects of hypothermia on energy metabolism in Mammalian central nervous system. J Cereb Blood Flow Metab. 2003;23(5):513-30. 
5. Nakashima K, Todd MM. Effects of hypothermia on the rate of excitatory amino acid release after ischemic depolarization. Stroke. 1996;27(5):913-8.

6. Xiong $\mathrm{M}$, Yang $\mathrm{Y}$, Chen GQ, Zhou WH. Post-ischemic hypothermia for $24 \mathrm{~h}$ in $\mathrm{P7}$ rats rescues hippocampal neuron: association with decreased astrocyte activation and inflammatory cytokine expression. Brain Res Bull. 2009;79(6):351-7.

7. Zhao H, Steinberg GK, Sapolsky RM. General versus specific actions of mild-moderate hypothermia in attenuating cerebral ischemic damage. J Cereb Blood Flow Metab. 2007;27:1879-94.

8. Annoni F, Donadello K, Nobile L, Taccone FS. A practical approach to the use of targeted temperature management after cardiac arrest. Minerva Anestesiol 2020. Online ahead of print.

9. Callaway CW, Tadler SC, Katz LM, et al. Feasibility of external cranial cooling during out-of-hospital cardiac arrest. Resuscitation. 2002;53:159-65.

10. Castrén $M$, Nordberg P, Svensson L, et al. Intra-arrest transnasal evaporative cooling: a randomized, prehospital, multicenter study (PRINCE: pre-ROSC intranasal cooling effectiveness). Circulation. 2010;122:729-36.

11. Garret JS, Studnek JR, Blackwell T, et al. The association with intra-arrest hypothermia and return of spontaneous circulation among individuals experiencing out of hospital cardiac arrest. Resuscitation. 2011;82:21-5.

12. Debaty $G$, Maignan $M$, Savary $D$, et al. Impact of intra-arrest hypothermia in outcomes of prehospital cardiac arrest: a randomized controlled trial. Intensive Care Med. 2014;40(12):1832-42.

13. Schenfeld EM, Studnek J, Heffner AC, et al. Effect of prehospital initiation of therapeutic hypothermia in adults with cardiac arrest on time-to-target temperature. CJEM. 2015;17(3):240-7.

14. Bernard SA, Smith K, Finn J, et al. Induction of therapeutic hypothermia during out-of-hospital cardiac arrest using a rapid infusion of cold saline: the RINSE trial (Rapid Infusion of Cold Normal Saline). Circulation. 2016;134:797-805.

15. Freese J, Hall CB, Lancet EA, et al. Intra-arrest induction of hypothermia via large volume ice-cold saline for sudden cardiac arrest: the New York City project hypothermia experience. Ther Hypothermia emp Manag. 2019;9(2):128-35. (ePub Nov 2018).

16. Nordberg P, Taccone FS, Truhlar A, et al. Effects of trans-nasal evaporative intra-arrest cooling on functional neurological outcome in out-of-hospital cardiac arrest. The PRINCESS randomized clinical trial. JAMA. 2019;321(17):1677-85.

17. Kim F, Nichol G, Maynard C, et al. Effect of prehospital induction of mild hypothermia on survival and neurological status among adults with cardiac arrest: a randomized clinical trial. JAMA. 2014;311:45-52.

18. Moher $D$, Shamseer $L$, Clarke $M$, et al. Preferred reporting items for systematic review and metaanalysis protocols (PRISMA-P) 2015 statement. Systematic Reviews. 2015;4:1-9.

19. Guyatt $\mathrm{GH}$, Oxman AD, Vist GE, et al. GRADE: an emerging consensus on rating quality of evidence and strength of recommendations. BMJ. 2008;336:924-6. 
20. Sterne JAC, Savović J, Page MJ, et al. RoB 2: a revised tool for assessing risk of bias in randomised trials. BMJ. 2019;366:14898.

21. Deeks JJ, Dinnes J, D'Amico R, et al. Evaluating non-randomized intervention studies. Health Technol Assess. 2003;7(27):27.

22. Arrich J, Holzer M, Havel C, et al. Pre-hospital versus in hospital initiation of cooling for survival and neuroprotection after out- of hospital cardiac arrest. Cochrane Database Syst Rev. 2016;3:CD010570.

23. Huang FY, Huang BT, Wang PJ, et al. The efficacy and safety of prehospital therapeutic hypothermia in patients with out-of-hospital cardiac arrest: a systematic review and meta-analysis. Resuscitation. 2015;96:170-9.

24. Diao M, Huang F, Guan J, Zhang Z, Xiao Y, Shan Y, Lin Z, Ding L. Prehospital therapeutic hypothermia after cardiac arrest: a systematic review and meta analysis of randomized controlled trials. Resuscitation. 2013;84:1021-8.

25. Hunter BR, O'Donnell DP, Allgood KL, Seupaul RA. No benefit to prehospital initiation of therapeutic hypothermia in out-of-hospital cardiac arrest: a systematic review and meta-analysis. Acad Emerg Med. 2014;21:355-64.

26. Nie C, Dong J, Zhang P, Liu X, Han F. Prehospital therapeutic hypothermia after out-of-hospital cardiac arrest: a systematic review and meta-analysis. Am J Emerg Med. 2016;34:2209-16.

27. Lindsay P, Buell D, Scales DC. The efficacy and safety of pre-hospital cooling after out-of-hospital cardiac arrest: a systematic review and a meta-analysis. Crit Care. 2018;22:66.

28. Scolletta S, Taccone FS, Nordberg P, et al. Intra-arrest hypothermia during cardiac arrest: a systematic review. Crit Care. 2012;16:R41.

29. Bruel C, Parienti JJ, Marie W, et al. Mild hypothermia during advanced life support: a preliminary study in out-of-hospital cardiac arrest. Crit Care. 2008;12:R31.

30. Kämäräinen A, Vikkunen I, Tenhunen J, et al. Induction of therapeutic hypothermia during prehospital CPR using ice-cold intravenous fluid. Resuscitation. 2008;79:205-11.

31. Yannopoulos D, Zviman M, Castro V, et al. Intra-cardiopulmonary resuscitation hypothermia with and without volume loading in an ischemic model of cardiac arrest. Circulation. 2009;120(14):1426-35.

\section{Tables}

Table 1: Summary of the studies comparing intra-arrest therapeutic hypothermia (IATH) to standard inhospital TTM (Controls). 


\begin{tabular}{|c|c|c|c|c|c|c|}
\hline $\begin{array}{l}\text { First Author, year } \\
\text { [ref] }\end{array}$ & $\begin{array}{l}\text { Type of } \\
\text { study }\end{array}$ & $\begin{array}{l}\text { Method for } \\
\text { IATH }\end{array}$ & $\begin{array}{l}\text { PATIENTS } \\
\text { (n) }\end{array}$ & $\begin{array}{l}\text { IATH } \\
\text { (n) }\end{array}$ & $\begin{array}{l}\text { Controls } \\
\text { (n) }\end{array}$ & $\begin{array}{l}\text { Outcome } \\
\text { Assessment timing }\end{array}$ \\
\hline $\begin{array}{l}\text { Callaway et al, } \\
2002 \text { [9] }\end{array}$ & $\mathrm{P}$ & $\begin{array}{l}\text { Cranial } \\
\text { cooling }\end{array}$ & 22 & 9 & 13 & Hospital Discharge \\
\hline $\begin{array}{l}\text { Castren et al, } \\
2010[10]\end{array}$ & $\mathrm{RCT}$ & TNEC & 194 & 93 & 101 & Hospital Discharge \\
\hline $\begin{array}{l}\text { Garrett et al,2011 } \\
\text { [11] }\end{array}$ & $\mathrm{R}$ & Cold fluids & 542 & 208 & 334 & Hospital Discharge \\
\hline $\begin{array}{l}\text { Debaty et al, } \\
2014 \text { [12] }\end{array}$ & RCT & Cold fluids & 245 & 123 & 122 & Hospital Discharge \\
\hline $\begin{array}{l}\text { Schenfeld et al, } \\
2015 \text { [13] }\end{array}$ & $\mathrm{R}$ & Cold fluids & 132 & 59 & 64 & Hospital Discharge \\
\hline $\begin{array}{l}\text { Bernard et al, } \\
2016 \text { [14] }\end{array}$ & $\mathrm{RCT}$ & Cold fluids & 1198 & 618 & 580 & Hospital Discharge \\
\hline $\begin{array}{l}\text { Freese et al, } 2018 \\
\text { [15] }\end{array}$ & $\mathrm{P}$ & Cold fluids & 492 & 313 & 179 & Hospital Discharge \\
\hline $\begin{array}{l}\text { Nordberg et al, } \\
2019 \text { [16] }\end{array}$ & $\mathrm{RCT}$ & TNEC & 668 & 337 & 334 & 3 months \\
\hline TOTAL & & & 3493 & 1760 & 1727 & \\
\hline
\end{tabular}




\begin{tabular}{|c|c|c|c|c|c|c|c|c|}
\hline \multirow[b]{2}{*}{$\begin{array}{l}\text { First Author, year } \\
\text { [ref] }\end{array}$} & \multicolumn{2}{|c|}{ ROSC } & \multicolumn{2}{|c|}{$\begin{array}{l}\text { Hospital } \\
\text { Admission }\end{array}$} & \multicolumn{2}{|c|}{ Survival } & \multicolumn{2}{|c|}{$\begin{array}{l}\text { Favorable } \\
\text { Neurological } \\
\text { Outcome }\end{array}$} \\
\hline & IATH & Controls & IATH & Controls & IATH & Controls & IATH & Controls \\
\hline $\begin{array}{l}\text { Callaway et al, } \\
2002 \text { [9] }\end{array}$ & 3 & 0 & 3 & 0 & 0 & 0 & 0 & 0 \\
\hline $\begin{array}{l}\text { Castren et al, } 2010 \\
\text { [10] }\end{array}$ & 35 & 43 & 33 & 42 & 14 & 13 & 11 & 9 \\
\hline $\begin{array}{l}\text { Garrett et al,2011 } \\
\text { [11] }\end{array}$ & 76 & 90 & 59 & 78 & 26 & 40 & NR & NR \\
\hline $\begin{array}{l}\text { Debaty et al, } 2014 \\
\text { [12] }\end{array}$ & 46 & 38 & 41 & 36 & 7 & 5 & 7 & 4 \\
\hline $\begin{array}{l}\text { Schenfeld et al, } \\
2015[13]\end{array}$ & NR & NR & NR & NR & 28 & 36 & 25 & 32 \\
\hline $\begin{array}{l}\text { Bernard et al, } 2016 \\
\text { [14] }\end{array}$ & 207 & 227 & 304 & 317 & 63 & 66 & 54 & 49 \\
\hline $\begin{array}{l}\text { Freese et al, } 2018 \\
\text { [15] }\end{array}$ & 167 & 101 & 118 & 80 & 33 & 32 & 15 & 19 \\
\hline $\begin{array}{l}\text { Nordberg et al, } \\
2019 \text { [16] }\end{array}$ & 183 & 152 & 143 & 140 & 60 & 52 & 56 & 45 \\
\hline TOTAL & 717 & 651 & 701 & 693 & 231 & 244 & 168 & 159 \\
\hline
\end{tabular}

$\mathrm{P}=$ prospective; $\mathrm{R}=$ retrospective; $\mathrm{RCT}=$ randomized controlled trial, $\mathrm{IATH}=$ intra-arrest therapeutic hypothermia; $T N E C=$ trans nasal evaporative cooling; $\mathrm{ROSC}=$ return of spontaneous circulation; $\mathrm{NR}=$ not reported

Table 2: Summary of the risk of bias (ROB) for randomized clinical trials comparing intra-arrest therapeutic hypothermia (IATH) with standard in-hospital targeted temperature management in adult outof-hospital cardiac arrest (OHCA) patients. 


\begin{tabular}{|c|c|c|c|c|c|c|}
\hline $\begin{array}{l}\text { First } \\
\text { Author, } \\
\text { year [ref] }\end{array}$ & $\begin{array}{l}\text { Randomization } \\
\text { process }\end{array}$ & $\begin{array}{l}\text { Deviation } \\
\text { from the } \\
\text { intended } \\
\text { interventions }\end{array}$ & $\begin{array}{l}\text { Missing } \\
\text { Outcomes } \\
\text { data }\end{array}$ & $\begin{array}{l}\text { Measurement } \\
\text { of the } \\
\text { Outcome }\end{array}$ & $\begin{array}{l}\text { Selection } \\
\text { of the } \\
\text { reported } \\
\text { results }\end{array}$ & $\begin{array}{l}\text { Overall } \\
\text { ROB }\end{array}$ \\
\hline $\begin{array}{l}\text { Castren } \\
\text { et al, } \\
2010 \\
{[10]}\end{array}$ & Low & Low & Low & Low & Low & Low \\
\hline $\begin{array}{l}\text { Debaty } \\
\text { et al, } \\
2014 \\
{[12]}\end{array}$ & Low & Low & Low & Low & Low & Low \\
\hline $\begin{array}{l}\text { Bernard } \\
\text { et al, } \\
2016 \\
{[14]}\end{array}$ & Low & Low & Low & Low & $\begin{array}{l}\text { Some } \\
\text { Concerns }\end{array}$ & $\begin{array}{l}\text { Some } \\
\text { Concerns }\end{array}$ \\
\hline $\begin{array}{l}\text { Nordberg } \\
\text { et al, } \\
2019 \\
{[16]}\end{array}$ & Low & Low & Low & Low & Low & Low \\
\hline
\end{tabular}

Table 3: Summary of risk of bias (ROB) for non-randomized clinical trials comparing intra-arrest therapeutic hypothermia (IATH) with standard in-hospital targeted temperature management in adult outof-hospital cardiac arrest (OHCA) patients. Low $\mathrm{ROB}=0$, high $\mathrm{ROB}=1$.

\begin{tabular}{|lllll|}
\hline First Author, year [ref] & $\begin{array}{l}\text { Selection of } \\
\text { cases }\end{array}$ & $\begin{array}{l}\text { Comparability of } \\
\text { cohorts }\end{array}$ & $\begin{array}{l}\text { Exposure and } \\
\text { outcome }\end{array}$ & $\begin{array}{l}\text { Overall } \\
\text { ROB }\end{array}$ \\
\hline $\begin{array}{l}\text { Callaway et al, 2002 } \\
\text { [9] }\end{array}$ & 1 & 0 & 0 & High \\
\hline $\begin{array}{l}\text { Garrett et al, 2011 } \\
{[11]}\end{array}$ & 1 & 0 & 0 & High \\
\hline $\begin{array}{l}\text { Schenfeld et al, 2015 } \\
\text { [13] }\end{array}$ & 0 & 1 & 0 & High \\
\hline $\begin{array}{l}\text { Freese et al, 2018 } \\
\text { [15] }\end{array}$ & 0 & 1 & 0 & High \\
\hline
\end{tabular}

\section{Figures}




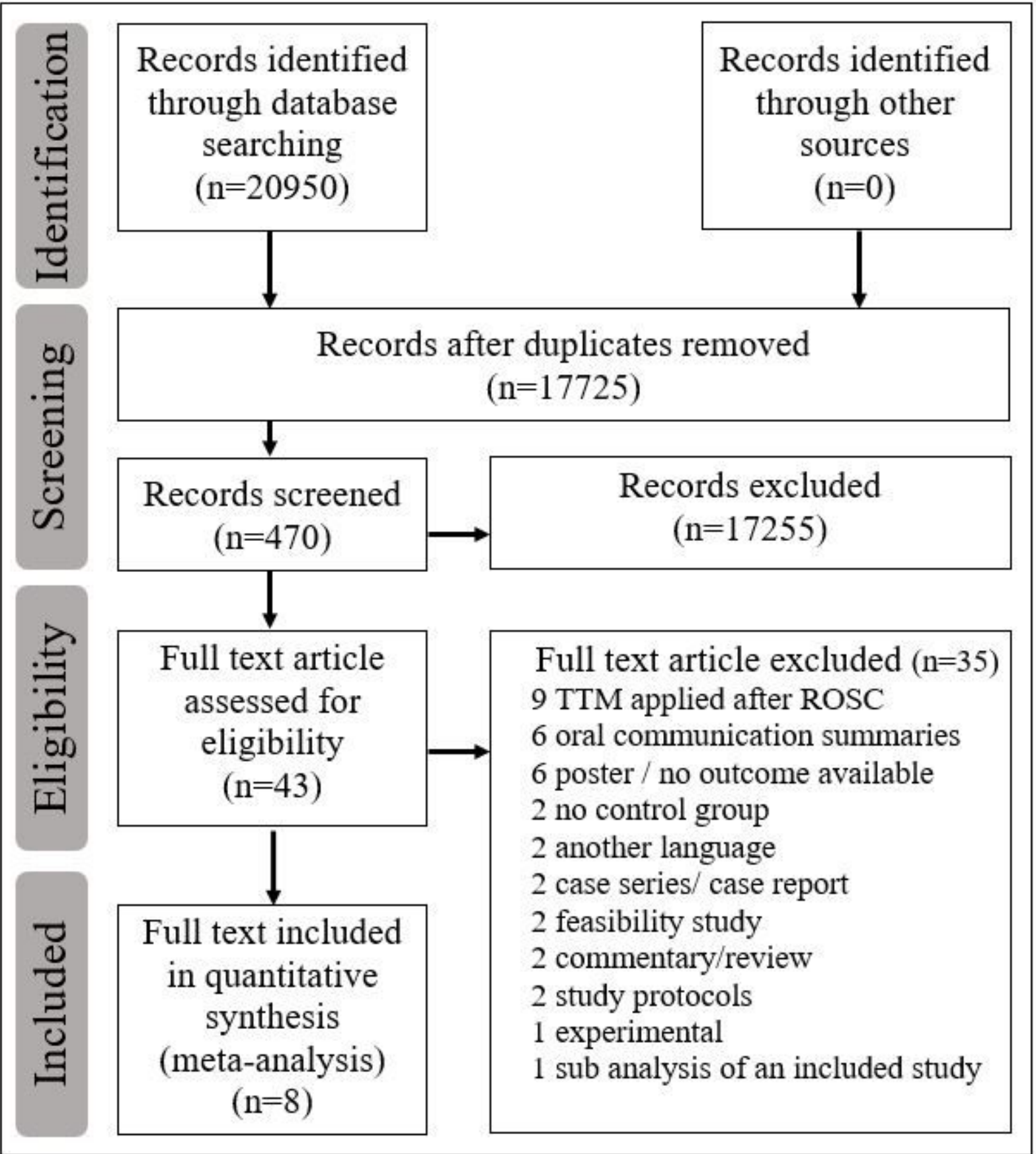

Figure 1

PRISMA-P flow diagram of the search results for original studies and selection of eligible trials. legend: TTM= Target Temperature Management, ROSC= Return Of Spontaneous Circulation 

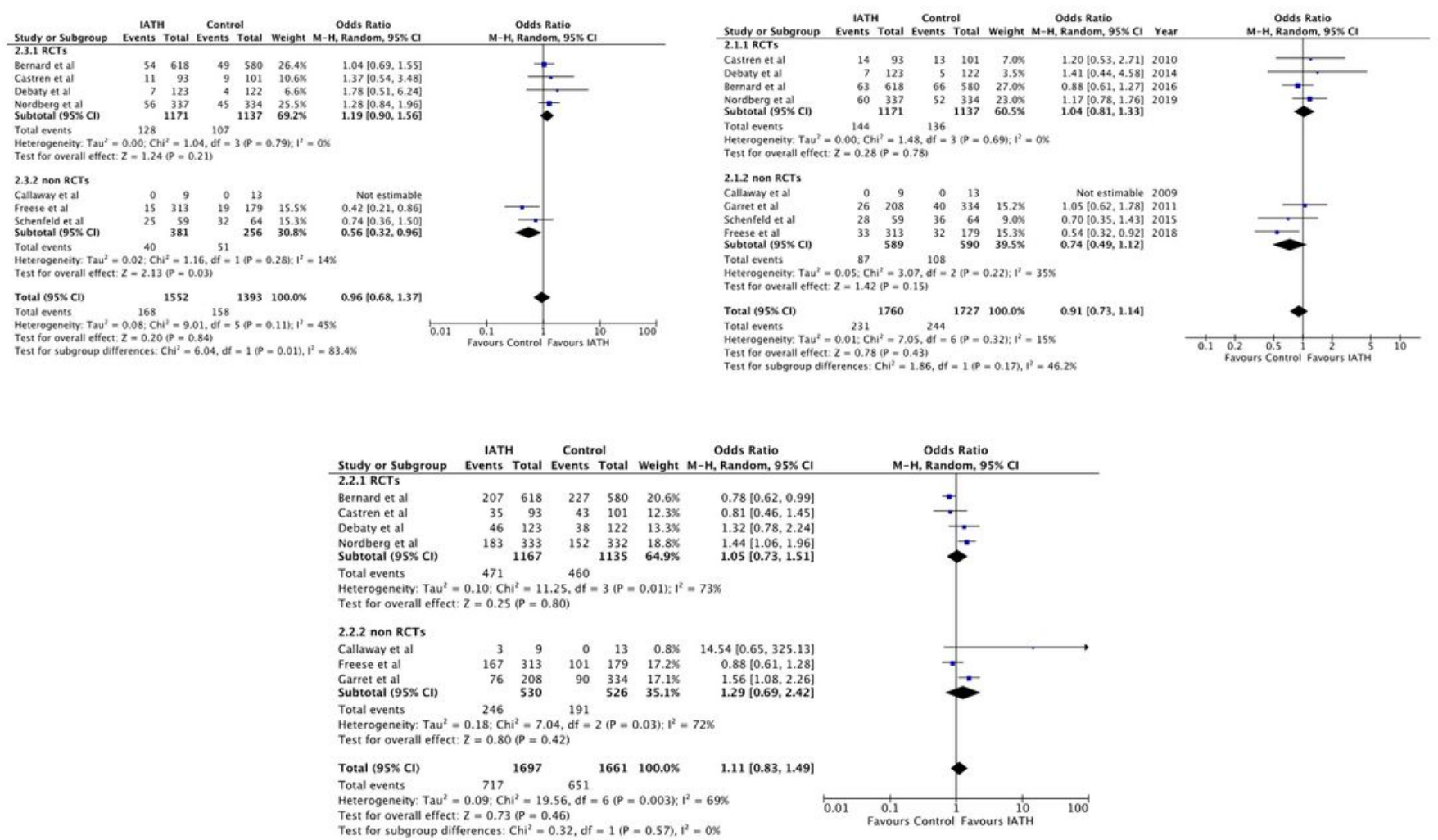

\section{Figure 2}

Forest plot for favorable neurological outcome (2A), return of spontaneous circulation (ROSC) rate (2B) and survival (2C) including randomized clinical trials (RCTs) and non-RCTs: intra arrest therapeutic hypothermia (IATH) vs standard in-hospital targeted temperature management (Control). legend: The size of the squares for the risk ratio reflects the weight of the trial in the pooled analysis. The horizontal bars represent $95 \%$ confidence intervals (Cls). 
IATH

Control

Odds Ratio

Odds Ratio

Study or Subgroup Events Total Events Total Weight $\mathrm{M}-\mathrm{H}, \mathrm{Random}, 95 \% \mathrm{Cl}$

3.3.1 TNEC

Nordberg et al

Castren et al

$\begin{array}{rr}56 & 337 \\ 11 & 93 \\ & 430\end{array}$

$45 \quad 334$

$41.1 \%$

$1.28[0.84,1.96]$

Subtotal $(95 \% \mathrm{Cl})$

67

9101

$8.6 \%$

$1.37[0.54,3.48]$

Total events 54

Heterogeneity: $\mathrm{Tau}^{2}=0.00 ; \mathrm{Chi}^{2}=0.02, \mathrm{df}=1(\mathrm{P}=0.89) ; \mathrm{I}^{2}=0 \%$

Test for overall effect: $Z=1.31(P=0.19)$

\subsubsection{Others}

Bernard et al

Debaty et al

Subtotal $(95 \% \mathrm{CI})$

$54 \quad 618$

$7 \quad 123$

$\begin{array}{rrr}49 & 580 & 45.5 \% \\ 4 & 122 & 4.7 \% \\ & 702 & 50.2 \%\end{array}$

$1.04[0.69,1.55]$

$1.78[0.51,6.24]$

Total events

61 53

Heterogeneity: $\mathrm{Tau}^{2}=0.00 ; \mathrm{Chi}^{2}=0.64, \mathrm{df}=1(\mathrm{P}=0.42) ; \mathrm{I}^{2}=0 \%$

Test for overall effect: $Z=0.45(P=0.66)$

Total $(95 \% \mathrm{Cl})$

Total events

1171 128 107

$1137100.0 \%$

Heterogeneity: $\mathrm{Tau}^{2}=0.00 ; \mathrm{Chi}^{2}=1.04, \mathrm{df}=3(\mathrm{P}=0.79) ; \mathrm{I}^{2}=0 \%$

Test for overall effect: $Z=1.24(P=0.21)$

Test for subgroup differences: $\mathrm{Chi}^{2}=0.38, \mathrm{df}=1(\mathrm{P}=0.54), \mathrm{I}^{2}=0 \%$
$1.19[0.90,1.56]$

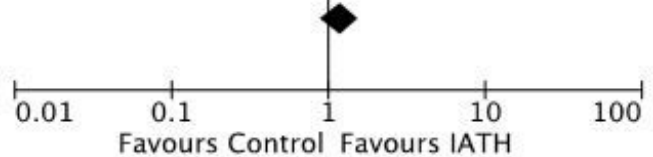

\section{Figure 3}

Forest plot for favorable outcome (FO) including randomized clinical trials (RCTs) using trans nasal evaporative cooling (TNEC) or other techniques (Others). Intra arrest therapeutic hypothermia (IATH) vs. standard in-hospital targeted temperature management (Control). legend: The size of the squares for the risk ratio reflects the weight of the trial in the pooled analysis. The horizontal bars represent $95 \%$ confidence intervals (Cls).

\section{IATH \\ Control \\ Odds Ratio}

Study or Subgroup

\subsubsection{Shockable}

Castren et al

Nordberg et al

Subtotal $(95 \% \mathrm{CI})$

Events Total

Weight $\mathrm{M}-\mathrm{H}$, Fixed, $95 \% \mathrm{Cl}$

Total events

$\begin{array}{rr}8 & 16 \\ 48 & 138 \\ & 154\end{array}$

$\begin{array}{rr}6 & 21 \\ 35 & 135 \\ & 156\end{array}$

$6.9 \%$

$61.8 \%$

$2.50[0.64,9.77]$

$1.52[0.91,2.56]$

56

41

Heterogeneity: $\mathrm{Chi}^{2}=0.44, \mathrm{df}=1(\mathrm{P}=0.51) ; \mathrm{I}^{2}=0 \%$

Test for overall effect: $Z=1.95(P=0.05)$

\subsubsection{Not Shockable}

$\begin{array}{lrrrrrr}\text { Castren et al } & 3 & 16 & 3 & 21 & 5.6 \% & 1.38[0.24,7.98] \\ \text { Nordberg et al } & 8 & 198 & 10 & 199 & 25.6 \% & 0.80[0.31,2.06] \\ \text { Subtotal (95\% CI) } & & \mathbf{2 1 4} & & \mathbf{2 2 0} & \mathbf{3 1 . 3 \%} & \mathbf{0 . 9 0}[\mathbf{0 . 3 9}, \mathbf{2 . 0 8}] \\ \text { Total events } & 11 & & 13 & & & \end{array}$

Heterogeneity: $\mathrm{Chi}^{2}=0.30, \mathrm{df}=1(\mathrm{P}=0.59) ; \mathrm{I}^{2}=0 \%$

Test for overall effect: $Z=0.24(P=0.81)$

Total $(95 \% \mathrm{Cl})$

368

$376 \quad 100.0 \%$

$1.40[0.92,2.12]$

Total events

67 54

Heterogeneity: $\mathrm{Chi}^{2}=2.15, \mathrm{df}=3(\mathrm{P}=0.54) ; \mathrm{I}^{2}=0 \%$

Test for overall effect: $Z=1.57(P=0.12)$

Test for subgroup differences: $\mathrm{Chi}^{2}=1.42, \mathrm{df}=1(\mathrm{P}=0.23), \mathrm{I}^{2}=29.7 \%$
Odds Ratio

$\mathrm{M}-\mathrm{H}$, Fixed, $95 \% \mathrm{CI}$

\section{Figure 4}


Forest plot for favorable neurological outcome (FO) in patients presenting with shockable rhythms vs non-shockable rhythm: intra arrest therapeutic hypothermia (IATH) vs. standard of care (Control). legend: The size of the squares for the risk ratio reflects the weight of the trial in the pooled analysis. The horizontal bars represent $95 \%$ confidence intervals (Cls).

\section{Supplementary Files}

This is a list of supplementary files associated with this preprint. Click to download.

- FigureS3.docx

- Figures2.docx

- Figures1.docx

- Appendix1.docx

- Tables1.docx 